\title{
The measurement of social performance in third sector organizations
}

\author{
[ J. D. Cabedo; I. Fuertes; A. Maset-Llaudes; J.M. Tirado ]
}

\begin{abstract}
Nonprofit organizations have developed a great deal of methods for measuring the social impact of their programs. In this paper we show an overview of them, underlying their advantages and drawbacks, to identify which ones are suitable for social performance information disclosure. In addition, we do a proposal on the scope of this disclosure.
\end{abstract}

Keywords-social impact, social performance measurement, information disclosure

\section{Introduction}

There is no doubt that nonprofit organizations and, in general, third sector organizations play a key role for solving or relieving a series of fundamental problems faced by mankind: healthcare, education, emergency situations, human rights, .... These organizations need funds to finance such social programs. And funders (large funders and anonymous citizens) want to know not only the final destiny of these funds, but also the utility of the actions undertaken with the provided funds. Some of these organizations are nonprofit or charitable organizations, whereas others (i.e. social entrepreneurs) make up an important social industry sector that similarly needs to show what the outcomes and impacts of their activities are.

The business world measures the performance of an organization in financial terms. But this approach is not useful for third sector organizations. Even if profitable activities are carried out, the financial performance is not a good benchmark, because profit is not their motivation. Their activity is focused on the solution of social problems and, therefore, using social performance is better than financial performance for the accurate measurement of their success.

In this paper we describe some of the methods used by organizations for measuring their social performance: Moreover, we analyse the advantages and drawbacks when they are used for different aims.

We have structured the rest of the paper as follows: in the second section we provide an overview of the main characteristics of the abovementioned methods, underlying their advantages and drawbacks; section three analyses the potential utilization of these methods when they are used for different aims; and section four summarizes the main conclusions of the paper

\section{J. D. Cabedo; I. Fuertes; A. Maset-Llaudes; J.M. Tirado}

Department of Finance \& Accounting / Universitat Jaume I

Spain

Corresponding author:

This research was supported by Generalitat Valenciana (Project: AICO/2015/024)

\section{Methods to assess the social impact of third sector organizations}

The academic literature on this topic is not vast. Furthermore, most of the methods that academic papers analyse are those previously developed and implemented by nonprofit organizations. Therefore, we are going to describe the main characteristics of the methods by sometimes using particular examples taken from those organizations that are actually using them.

A first classification of these methods distinguishes between methods that solely calculate a rate for the social impact of the activity or activities carried out, and methods that use other resources. The former emulate the procedure to estimate a corporation's financial performance and, therefore, use a monetary approach. The latter focus mainly on non-monetary indicators. According to Ebrahim and Rangan [1] these methods can be classified in the following categories:

- $\quad$ Experimental methods

- Logic methods

- $\quad$ Strategy approaches

- Participatory and relationship-based methods

\section{A. Experimental methods}

These methods are based on randomized control trials. The Abdul Latif Jameel Poverty Action Laboratory (see its methodology at http://www.povertyactionlab.org/) underlines the utility of them for clinical trials, agricultural experiments and social programs (social experiments). The social impact of an activity or a set of activities is measured in the following way (as a matter of fact, this is the methodology used in medical essays): the program is implemented on a set of individuals, called treatment group, randomly selected (you can randomly select the individuals, the households, the area, ... where the program must be implemented). Simultaneously, another set of individuals (called control group) must be randomly chosen; the program will not be implemented on this group. After a time period, the results reached in the treatment group must be compared against those obtained in the control group. These results are usually measured through the proportion of individuals, households, ... that, after this period, have improved their situation in the direction pursued by the implemented program. The difference between the results of both groups will provide a measure of the impact of the program. 
When implemented, this methodology involves additional problems (spillovers, crossovers, ....). But basically it works as stated above. Its main advantage: the method is statistically precise. But it has an important drawback: it can be only used on those programs whose results can be appreciated in a medium time period. It is not suitable for long term impacts. Another drawback stems from the difficulties for comparing the results reached by different kinds of programs.

\section{B. Logic methods}

The W.K. Kellogg Foundation [2] uses logic models to evaluate its programs. A logic model is, basically, a systematic and visual way to show the relationships among the resources invested in the program, the activities that this program involves, and the expected changes or results. Figure 1 show a diagram for a basic logic model. Actual models are usually more complex than the one showed in Figure 1, but the underlying idea is the same for all of them: providing a road map of the forecasted resources, outcomes and impacts of a program.

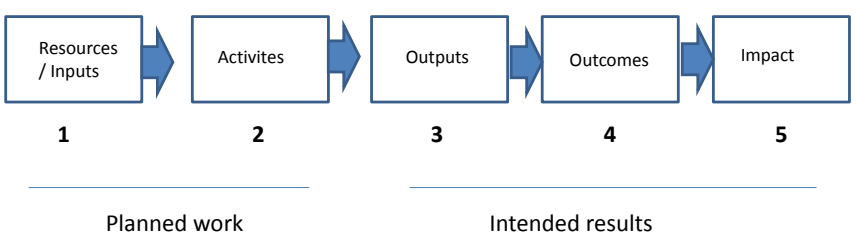

Figure 1. A basic logic model (Source: W.K. Kellog Foundation [2])

Most of the elements of a logic model are usually displayed in qualitative terms. It helps stakeholders to understand how the program works and where it is going to. Probably a logic model is the best way of depicting the program as a whole: it is a comprehensive, intuitive and clarifying tool. But this tool mainly works for visualising programs one by one. It is difficult to use logic models when we wish to compare different programs, with different outcomes and different impacts. For example, it is hard to compare the results reached by a program focused in fighting against diarrhoea in communities that drink contaminated water with those reached by a program focused on providing micro credits to entrepreneurs of a community. The results of the former will be probably measured through a decrease in disease incidence at the communities, whereas the results of the latter will be probably measured through the impact on the income of the community. The heterogeneity of the impacts brings about a difficulty for their comparison. This can be pointed out as the main drawback of the logic models.

\section{Strategy approaches}

Dashboards and Balanced Scorecards are two management tools, widely used in the corporations' field, which some nonprofit organizations have incorporated into their procedures.

As Bell and Masaoka [3] state, a nonprofit dashboard gives important information to decision makers such as executives and boards in a quick-read way. It turns on signal lights (red, yellow and green) and it can also display trend lines. But a dashboard is not a method for measuring the social impact of a program or an organization. It is management tool where the measurement of social impact can be incorporated as an item.

In the corporations' field, the use of Balanced Scorecards involves the assessment of an organization not only through its financial performance, but through other perspectives like customer, internal and innovation \& learning perspectives. For nonprofits, Zimmerman [4] proposes a balanced scorecard based on six categories (perspectives):

- $\quad$ Revenue and funding

- Resource allocation

- Donors and Board members

- Product and service recipients

- Internal operations

- Staff development

Probably this list lacks of an important category: the social impact. But, as it occurs with dashboard, a balanced scorecard is also a management tool. It is not a method to measure the social impact of a program.

\section{Participatory and relationship- based methods}

Methods like the Most Significant Changes Approach (MSC), the Participatory Poverty Assessment (PPA), the Participatory Rural Appraisal (PRA) and the Outcome Mapping can be included within this group.

The Outcome Mapping is a comprehensive tool for designing, monitoring and evaluating programs. Figure 2 shows the three stages of this method.

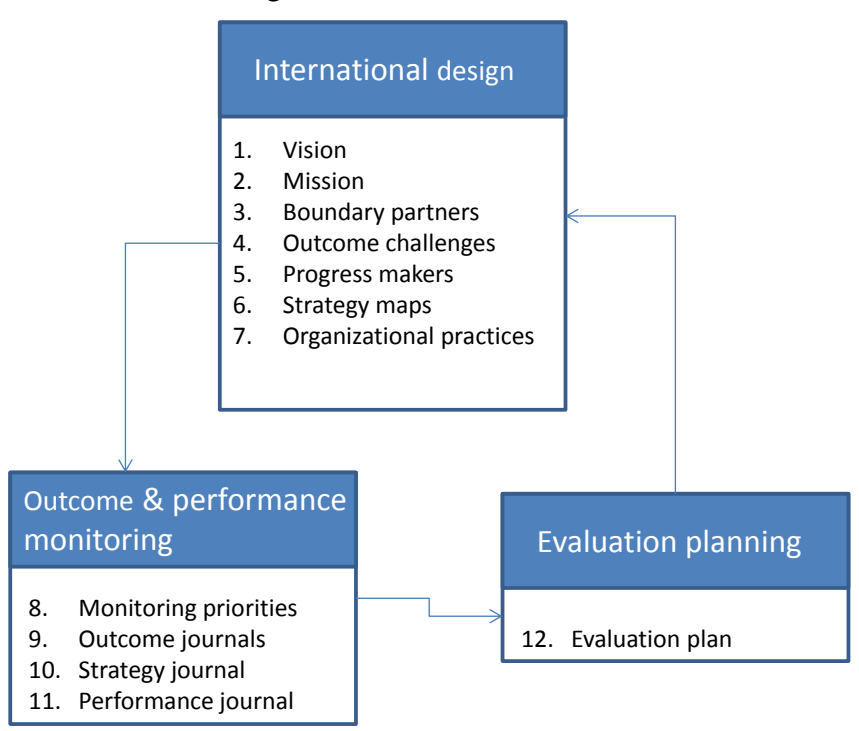

Figure 2. Three stages of the Outcome Mapping (Soruce: Earl et al. [5])

The PPA (see Norton et al. [6]) is an instrument for including poor people's views in the analysis of poverty and the formulation of strategies to reduce it through public policy. The PPA is generally carried out as policy research exercises, linked to governmental policy processes, aimed at understanding poverty from the perspective of poor people. Therefore it is not a method for, directly, measuring the social impact or the social performance of a program. The 
information collected from the PPA participants can be used for the measurement of the social performance, and this measurement has an undoubtable advantage: the potential beneficiaries are those who report about the impact of the program. But it has also some drawbacks. The PPA is mainly designed as a tool for improving the design of future programs through the feedback. Many of the social impact measurements can be qualitative ones and, therefore, they cannot be used for comparison purposes. Moreover, PPA is focused on poverty, a very important matter but it is not the only one boarded by third sector organizations.

MSC methods (see Davies and Dart [7]) choose the most significant change induced by a program through a series of meetings where the participants involved in the program shall jointly decide on the most remarkable change reached. It has the same advantage that the PPA had: The beneficiaries decide about the impact of the program. But it also has a drawback: the most significant change is expressed only in qualitative terms. Therefore, this measure of social performance or social impact for a program is not easily comparable with the results provided by other programs.

The PRA method (see Chambers [8]) introduces the participation through experiments.

\section{E. Methods based on a rate of return}

The methods of this group calculate a rate of return by comparing the outcomes reached through a program with the resources allocated in the program. This comparison is made in monetary terms. Therefore, all the outcomes must be monetised in order to calculate the rate of return. In this group the most used methods are the following ones: Best Available Charitable Option (BACO), Economic Rate of Return (ERR), Benefit Cost Ratio (BCR) and Social Return on Investment (SROI).

The main difference stems from how each method calculates the monetary value of the outcomes:

The BCR, used by the Robin Hood Foundation [9], assesses the direct improvement on the beneficiaries' income of the activities undertaken within an action program. The advantage of this method is its ease of comparing alternative programs. Its main drawback: its limited scope; as it depends on incomes, those programs with no direct impact on incomes are no easily measurable.

The Acumen Fund [10] uses the BACO method for evaluating the performance of its loans. This method assesses, as a first step, the social impact of the activities carried out within an action program. As not all the impacts are financial ones, it is necessary (in this stage) the monetization of some of the outcomes provided by the program. Only the financial (monetary) value of the direct impacts are taken into account. In a second step, the rate of return calculated from this value, is compared with the rate of return provided by other (alternative) charitable options. This comparison is used to decide about the program. Similarly to the BCR, the advantage of the method also arises from the easy way that it provides to compare alternative programs. Its main drawback: its limited scope; in some fields we can find no charitable option to use as benchmark.
The ERR [11] calculate the impact of the undertaken programs in macro-magnitudes. It can be very convenient to assess the impact at country level. But it does not fit in the evaluation of the social performance of organizations or narrower programs.

Finally, the SROI (see Cabedo and Fuertes [12]) is probably the most comprehensive methodology for calculating the rate of return of a social investment. It involves the monetisation of all the outcomes and impacts provided by a program. The SROI includes so the direct as the indirect outcomes of the program. The advantages: it provides a right estimation of the actual rate of return, and the methodology also involves the participation of all the relevant agents. The drawbacks: apart from the monetisation problems shared with preceding methods of this group, the need to quantify not only the direct impacts but also the indirect ones is a major shortcoming. The latter requires to mamke a series of subjective assumptions that can bias the results provided by the method

\section{Potential utilization}

In the preceding section we have summarized the main characteristics of the methods that third sector organizations are using for measuring their social performance. One can think that it is hard to answer the question about which one can be considered to be the best method. But nevertheless it is easy. All of them are perfectly valid. Think about a similar question that can be asked in the corporations' field: which of the methods used in banking to evaluate potential loans is the best one? The Societe Generale methodology? The Deutsche bank methodology? ... The answer is straightforward. All of them are valid. Surely, if we analyse some of them, we will find advantages and drawbacks, as we have detected in the preceding section. But all of them have been designed for helping analysts in their decision making process. In the case of banking, to help analysts to make a decision about whether to give a loan or not. In the field of nonprofit organizations, to help decision agents to make decisions on a program with social impact.

For analysing and deciding about programs, each organization must design, implement and improve its own methodology. This must be adapted to the characteristics and needs of the organisation. i.e. the Robin Hood Foundation focuses its programs in fighting against the poverty in New York city. This organization needs an evaluation tool that fits its particular requirements. And probably the BCR methods meet most of them. This methodology allows the organization to easily compare between non-homogeneous programs. However we can find other organization which does not need this kind of comparison. In this case, other evaluation methodology can be used even if it does not allow easy comparisons.

In summary, in order to analyse projects or programs, each organization not only can but must use its own methodology. It will be an internally directed tool and, therefore, must meet all the requirements of the internal users.

A different question is the one that arises when we seek a method that can measure the social performance or social impact in a homogeneous way, in order to compare the results reached by different organizations. It is an externally 
directed issue, and therefore, it must meet the requirements of the stakeholders, in the widest sense of the word.

The method used for this aim must provide a measure for the social performance with similar characteristics regardless the organization. Otherwise comparison will not be possible. As we have shown in the preceding section, only the methods belonging to the last group (the ones based on a rate of return) can meet this requirement. Therefore, for disclosing purposes, it would be advisable that third sector organizations calculate a rate of return for their social impact.

Is the disclosure of the social impact important? No, it is fundamental. In all the documents related to the measurement of social impact we will find a reference to the need of this kind of infomation for transparency purposes; to meet the information requirements of the stakeholders. The remaining question now is the one about the best method for this purpose. We have seen four of them. We have underlined the limited scope of the ERR. This method must, therefore, be dismissed for a general disclosing framework. We have also highlighted the difficulties that we can have when finding charitable options for some of the activities undertaken by third sector organizations. Furthermore, the decision about the best charitable option to use as a benchmark is clearly subjective. Therefore this method is not suitable for the purposes we are discussing.

Thus, only two methods (BCR and SROI) remain as the suitable ones for calculating a rate of return for the social performance for disclosing purposes. Basically both of them use the same calculation procedure: numerator, the monetary net present value of the outcomes; denominator, the resources invested for reaching these outcomes. Both methods also face similar problems when calculating the monetary equivalents of these outcomes (what is known as the monetisation process). Perhaps the main difference between them stems from the degree with which each agent participation is included. The SROI methodology is a participative one, whereas BCR provides a procedure for a social rate of return calculation, regardless the data come from. Other difference is the comprehensive character of the SROI method: it encompasses so the direct as the indirect monetary values of the outcomes. The BCR mainly focuses on the monetary value of the outcomes that can be directly associated to a program.

The comments we have just done do not mean that SROI is better than BCR. The SROI calculation procedure requires time and resources. On the contrary, BCR calculation can be easier. Therefore, it could be advisable to use the latter when only direct outcomes must be considered. Otherwise, SROI is the most suitable method.

At this point, a fundamental question to solve is whether the nonprofit or third sector organizations must report about their social impact considering only the direct outcomes induced by their activities or also considering the indirect ones. The implementation of SROI methodology requires a great deal of resources that many organizations cannot afford. But reporting only direct impacts depicts an incomplete view of the actual impact of the organization actions.

One possibility for solving the question could be the requirement of two tiers of disclosure. A compulsory tier, where organizations must calculate and disclose a rate of return for the social impact considering only the direct outcomes induced by their programs and activities. And a voluntary tier, where the information provided would be a comprehensive measure of the social impact of the organization. Thus, all the organizations would disclose a measure of the social impact calculated by assuming similar hypothesis and by using similar methodologies. This measure would allow the comparison among all organizations. On the other hand, those organizations whisking to reveal information about the actual social impact of their activities, could also disclose an additional rate for their social performance. This would allow a second level of comparison, restricted to the organizations that, voluntarily, disclose this information.

\section{Iv. Concluding remarks}

Third sector organizations are performing day by day a more important role in our society. The stakeholders and, in general terms, the society, need to know the impact of the activities of these organizations. This impact cannot be measured in financial terms. It must be measured under social parameters.

The organizations have developed a great deal of methods for measuring the social impact of their programs. In this paper we have shown an overview of them, underlying their advantages and drawbacks. All this methodologies are valid for evaluating and monitoring the programs. None of them can be considered to be the best one.

However if we want to compare the social performance between different organizations involved in different programs, a standardised social performance measure is required. Only the methods that calculate a rate of return for the social performance must be used at this point. Among them, the Social Return on Investment (SROI) and the Benefit Cost Ratios (BCR) are the most suitable ones.

Another important question is the scope of the information on social impact disclosed by the organizations. We propose a double tier disclosing framework: a first compulsory disclosure tier where, only direct outcomes are to be considered for the social performance measurement; and a second voluntary disclosure tier where organization could disclose any other measurement for the social performance by considering all the outcomes induced by their programs.

Anyway, the questions we have presented here are not closed. Additional research, mainly in the academic area, would be advisable.

\section{References}

[1] A. Ebrahim and V.K. Rangan, The limits of Nonprofit impact: a contingency framework for measuring social performance. Working Paper 10-099. Harvard Business School, 2010

[2] W.K. Kellogg Foundation, Logic Model development guide: Using Logic Models to bring together planning, evaluation and action. W.K. Kellogg Foundation, Michigan (USA), 2004.

[3] J. Bell and J. Masaoka, "A nonprofit dashboard and signal light for boards". Blue Avocado, July, 2009

[4] J. Zimmerman, Using a Balanced Scorecard in a Nonprofit Organization. CDR White Paper Collection of the Nonprofit Learning Center, Creative Direct Response Inc., Crofton, MD (USA), 2004 
Proc. of The Third Intl. Conf. on Advances in Social Science, Management and Human Behaviour - SMHB 2015

Copyright (c) Institute of Research Engineers and Doctors, USA .All rights reserved.

ISBN: 978-1-63248-067-5 doi: 10.15224/ 978-1-63248-067-5-68

[5] S. Earl, F. Carden and T. Smutylo, Outcome Mapping. Building Learning and Reflection into Development Programs. International Development Research Centre, Ottawa, ON (Canada), 2001

[6] A. Norton, B. Bird, K. Brock, M. Kakande and C. Turk, A rough guide to PPAs. Participatory Poverty Assessment. An introduction to theory and practice. Overseas Development Institute (UK), 2001

[7] R. Davies and J. Dart, The Most Significant Change' (MSC) Technique. A guide to its use. http://www.mande.co.uk/docs/MSCGuide.pdf (site visited: 15 August 2015), 2005

[8] R. Chambers, "Participatory Rural Appraisal (PRA): Challenges, Potential and Paradigm", World Development, Vol 22 (10), pp. 14371454, 1994

[9] M.M. Weinstein and C. Esposito-Lamy, Measuring Success: How Robin Hood estimates the Impact of Grants, Robin Hood Foundation, New York, NY (USA), 2009

[10] Acumen Fund, The Best Available Charitable Option, (site visited: 3 August 2015), 2007

[11] Millennium Challenge Corporation, Compact Development Guidance, (site visited: 15 August 2015), 2013

[12] J. D. Cabedo and I. Fuertes, "Social return and financing of urban regeneration policies", 51th Congress of the European Regional Science Association and 37th Meeting of Regional Studies of the Spanish Association of Regional Science. Proceedings. Paper 1690, Barcelona (Spain), 2011 